\title{
The role of Perioperative Surgical Home on health and longevity in society: importance of the surgical prehabilitation program
}

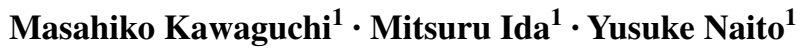

Received: 28 January 2017 / Accepted: 18 February 2017 / Published online: 3 March 2017

(C) Japanese Society of Anesthesiologists 2017

\section{Introduction}

Regardless of recent advances in surgical techniques, improvements in perioperative management remain crucial to avoid postoperative functional disability and complications, especially in an increasingly aged population. The development of perioperative complications associated with prolonged hospitalization and reduced functional capacity after major surgery may affect a patient's ability to return to daily living without any dependence. Therefore, in order to accomplish health and longevity in society, perioperative management is an important issue that needs to be resolved. A number of approaches to provide better postoperative recovery and outcome, including preoperative, intraoperative and postoperative strategies, have been applied by multidisciplinary teams [1-4]. A recent model called 'Perioperative Surgical Home' (PSH) has been established which enables surgical patients to be consulted from the time when the surgeon decides to operate and provides preoperative intervention during the waiting time for surgery. In this review, we focus on preoperative management to improve functional capacity, termed 'prehabilitation', as a recent approach for surgical patients $[5,6]$.

\section{Healthy life expectancy}

Over the past century, the average years of life (life expectancy) have been continuously increasing in most

Masahiko Kawaguchi

drjkawa@naramed-u.ac.jp

1 Department of Anesthesiology, Nara Medical University, 840 Shijo-cho, Kashihara, Nara 634-8522, Japan countries. In Japan, the average life expectancy in 2015 was 80.79 years for men and 87.05 years for women. In 2015, Japanese people aged $\geq 65$ years comprised $26.7 \%$ of the entire population; this figure is estimated to reach $37.1 \%$ in 2060 (both figures are the highest in the world (data from White Paper on the Aging Society FY 2015, Cabinet Office, Government of Japan, 2016). While an extension of average life expectancy is desirable, the important issue is an extension of healthy life expectancy, which is the period in the later years of life where the elderly are not bedridden or physically impaired. Although both life expectancy and healthy life expectancy have been increasing, the gap between them has also been widening. The healthy life expectancy in Japan was reported to be 71.1 years for men and 75.6 years for women in 2013, while the gap between life expectancy and healthy life expectancy was 8.9 years (11.2\%) for men and 10.8 years $(12.5 \%)$ for women [7].

According to an analysis of long-term care insurance in Japan, the common conditions for which long-term care services are required by individuals aged $>65$ years include cerebrovascular disorders, dementia and problems related to the locomotive organs, such as falls/fractures, joint disorders and aging frailty [8]. Therefore, in addition to the development of disease, frailty and locomotive organ disorders involving musculoskeletal dysfunction are major problems among the elderly which can affect mobile function, activities of daily living and quality of life.

\section{Locomotive syndrome and frailty}

The Japanese Orthopedic Association proposed the concept of 'locomotive syndrome', which is a condition requiring nursing care services, or high-risk conditions that will require such services soon because of locomotive organ 
dysfunction in the elderly $[9,10]$. The weakness of locomotive components, consisting of bones, joints, muscles and nerves, causes difficulty in standing, walking, running, climbing stairs, and performing other physical functions essential to daily life. Sarcopenia, one of the components of locomotive syndrome, is diagnosed based on both low muscle mass and low muscle function (strength or performance).

Frailty is an age-related syndrome characterized by decreased reserve and resistance to stressors and classified into physical, social and cognitive frailty, all of which increase the risk of adverse health outcomes, such as falls, disability, long-term care, and mortality [11, 12]. The terms of frailty and locomotive syndrome are similar, but locomotive syndrome is specified as physical frailty with musculoskeletal disease without social or cognitive frailty (Fig. 1) [13]. The associations among full performance (health), frailty and disability are shown in Fig. 2. In older adults with frailty, locomotive syndrome with sarcopenia more likely represent the consequences of a permanent disruption of homeostasis, and is placed between robust health and disability, regarded as a pre-disability stage.

\section{Prevention of locomotive syndrome and frailty}

There have been a number of studies that indicated the effectiveness of physical intervention in limiting the disability and functional decline of mobility, strength, balance and gait in an aged population with mild to moderate disability [9]. Since its efficacy is limited in people with severe disability, early detection of the locomotive syndrome and early intervention have been advocated. Physical interventions are based on exercise, and locomotion training can be

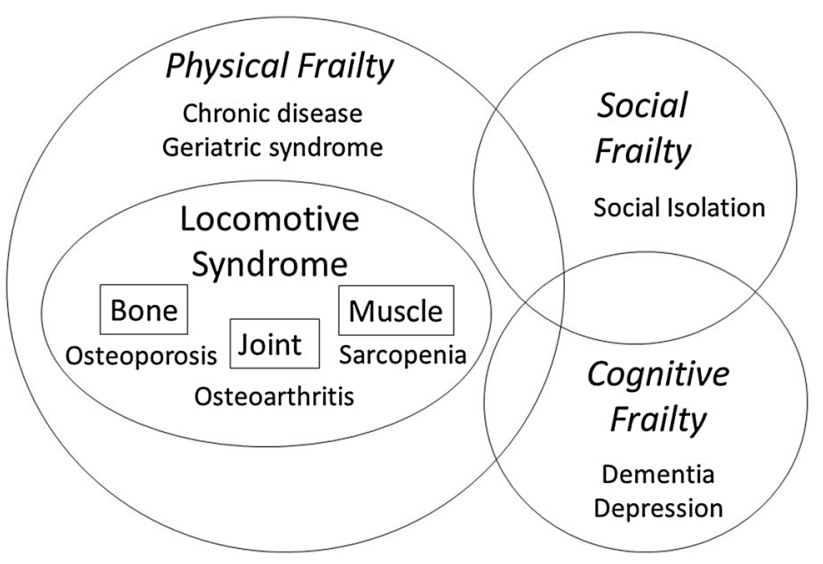

Fig. 1 Associations among the components of frailty and locomotive syndrome. Frailty is classified as physical, social and cognitive frailty. Locomotive syndrome is placed in physical frailty, and consists of weakness of bones, joints and muscles (nerve)

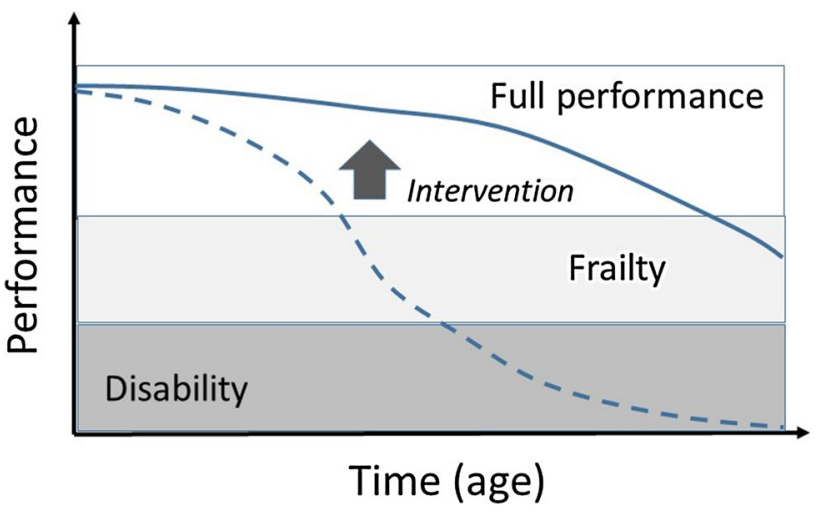

Fig. 2 Associations among full performance (health), frailty and disability with aging. Frailty is placed between full performance and disability. The time when frailty develops may be delayed by intervention

applied to improve balance and strengthen muscles. These exercises include single-leg standing with eyes open, squatting, walking and other exercises such as heal raising and front lunges. Locomotive training is considered to be effective in improving physical function and quality of life in the elderly.

Rizzuto et al. [14] investigated the modifiable factors associated with longevity among 1810 adults aged $\geq 75$ years with follow-up for 18 years, and indicated that lifestyle behaviors such as not smoking and physical activity were associated with longer survival. Of the leisure activities, physical activity was most strongly associated with survival. The median age at death of participants who regularly swam, walked, or performed gymnastics was 2 years higher than those who did not. The median survival of people with a low risk profile (healthy lifestyle behaviors, participation in at least one leisure activity, and a rich or moderate social network) was 5.4 years longer than those with a high-risk profile. Shinkai et al. [11] examined the impact of 10 -year community intervention to promote physical activity, nutrition and social participation by means of a health education program on healthy aging among older adults aged $\geq 65$ years, and demonstrated that such an approach was effective in preventing frailty and promoting healthy aging.

\section{The impact of surgery on the elderly}

Major surgery is associated with pain, sleep dysfunction, fatigue, decreased physical activity and deconditioning as well as the development of complications. Hospitalization following surgery can further reduce physical activity and exacerbate deconditioning, especially in elderly patients. Even in the absence of complications, there is often a 
$20-40 \%$ reduction in physiologic and functional capacity after major surgery [15]. Affected patients can develop temporary or permanent dependence in their activities of daily living, which prolongs the hospital length of stay and subsequently increases the risk of complications and morbidity, and may reduce the quality of life. These situations resemble locomotive syndrome and frailty in the elderly and hospitalized patients undergoing surgery can be considered to have a high risk of locomotive syndrome and frailty. In addition, the development of delirium and cognitive dysfunction after surgery further deteriorates the patient's physical status due to increased morbidity and hospital length of stay, especially in elderly patients $[16$, 17].

Preoperative functional capacity has been reported to be an important predictor of postoperative outcome [18, 19]. Functional capacity reflects underlying physiological reserve, indicating the ability to endure the physiological stress of major surgery and the harmful effects of prolonged postsurgical bed rest and poor perioperative nutritional intake. Functional capacity can be assessed using exercise tests and, therefore, exercise capacity is often used to estimate perioperative risk regarding postoperative recovery. In addition to exercise capacity, there are modifiable physical and behavioral factors associated with postoperative morbidity, mortality and recovery, which include a patient's nutritional status, hemoglobin level, smoking, excessive alcohol, and psychological state such as anxiety and depression $[5,6]$.

\section{What is surgical prehabilitation?}

Traditionally, interventions to decrease perioperative complications and improve functional recovery have been provided as in-hospital or postoperative care. However, it has been advocated that the preoperative period is an ideal time to increase physical reserves to overcome surgical stress (Fig. 3). In particular, the waiting time from the decision on surgery until the date of surgery can provide an important window to optimize a patient's condition. The time window can vary depending on the condition of the patient and the hospital, but it may last for $>1$ month. Optimizing physical activity and function preoperatively can be an effective intervention to reduce perioperative morbidity, decrease the length of hospital stay and facilitate the patient's return to normal life, which is termed 'prehabilitation' [5, 6]. Prehabilitation is therefore defined as a strategy to support the patient in order to enhance their functional capacity and enable them to withstand the stress of surgery and associated inactivity.

Risk factors for a poor outcome after surgery can be divided into fixed factors and potentially modifiable factors.

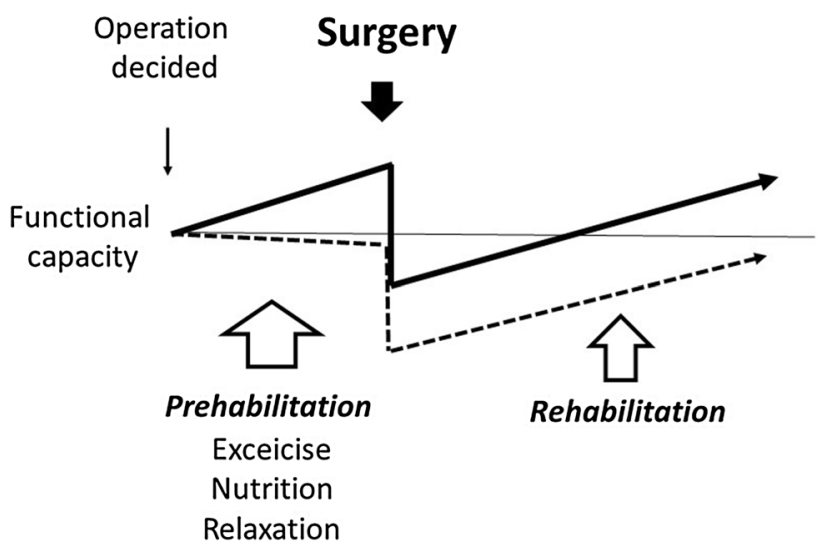

Fig. 3 A theoretic model of perioperative changes in functional capacity. Prehabilitation can improve functional capacity before and after surgery

Fixed factors include age and chronic comorbidities, for which careful management is required perioperatively. In contrast, modifiable factors for a poor outcome include preoperative physical activity level, smoking and alcohol consumption, which can be modified by the intervention and involvement of patients in the preoperative program. The prehabilitation program is a multimodal approach to target such modifiable risk factors and consists of exercise, nutritional support, psychological support and behavioral modification.

\section{Preoperative exercise training}

Exercise training during the preoperative period, consisting of inspiratory muscle training, aerobic exercise, and resistance training, has been the main feature of prehabilitation, in order to enhance the physiological reserve and facilitate postoperative recovery. Respiratory rehabilitation includes deep-breathing exercises, thoracic physiotherapy, incentive spirometry and inspiratory muscle training. Inspiratory muscle training is aimed at increasing inspiratory muscle strength and endurance by applying a resistive load to the inspiratory muscles. A recent Cochrane systematic review on inspiratory muscle training demonstrated that application of inspiratory muscle training reduced length of stay, reduced postoperative pulmonary complications, and reduced basal atelectasis [20].

Aerobic exercise combined with resistance training is used as a preoperative exercise intervention, although there is a variety of methodologies regarding the type of exercise used and the duration, frequency and timing of exercise training. The American Cancer Society has published a broad exercise guideline that recommends at least $150 \mathrm{~min}$ of moderate or $75 \mathrm{~min}$ of vigorous intensity (or combination of) exercise per week and includes $2-3$ sessions of 
resistance training, involving major muscle groups [21]. For example, a certified kinesiologist assessed and trained each participant to perform home-based training, consisting of aerobic, resistance and flexibility exercise up to 50 min three times per week [22, 23]. For aerobic exercise, cycling, walking, or swimming is used based on the physical capacity of each patient, assessed using the 6-min walking test distance. For resistance training, exercise targeting major muscle groups is performed with elastic bands. Further study would be required to decide which exercise program would be appropriate as a prehabilitation program.

\section{Nutritional prehabilitation}

Patients who are malnourished preoperatively have been shown to have a greater risk of morbidity and mortality, associated with longer hospital length of stay. Surgical stress can induce proteolysis and lipolysis to provide precursors for gluconeogenesis and a state of insulin resistance. This hypermetabolic state can result in significant protein loss, primarily from muscles, and a consequent reduction in functional capacity, persistent fatigue and slow postoperative recovery. Therefore, early detection of malnourished patients and consequent nutritional intervention are importantly applied. Nutritional intervention is performed to optimize nutrient stores preoperatively and provide adequate nutrition to compensate for this postoperative catabolic state.

In addition, nutritional intervention with protein supplementation can enhance preoperative exercise training, resulting in an improvement of preoperative functional capacity. Therefore, nutritional intervention can be applied to all patients who undergo a prehabilitation program with exercise training, regardless of preoperative nutritional condition [5, 6]. For example, a registered dietician estimates macronutrient and energy intake from each patient's food record, and discusses dietary goals with each patient. Patients can be provided protein supplementation to reach a daily intake of up to $1.2 \mathrm{~g}$ of protein $/ \mathrm{kg}$ of body weight, as per the European Society of Parenteral and Enteral Nutrition guidelines for surgical patients [22-24]. Protein supplement is recommended to be ingested within $1 \mathrm{~h}$ post exercise (anabolic window).

\section{Smoking cessation}

Smoking is associated with a reduced oxygen carrying capacity due to carbon monoxide, sympathomimetic effect, impaired immune function, impaired collagen production, reduced lung capacity, increased mucus production and impaired ciliary function [5]. All of these can lead to an increased incidence of complications, including wound infection, delayed healing and cardiopulmonary complications, and mortality after surgery. Smoking cessation before surgery is therefore an important clinical challenge to improve postoperative outcomes. A recent Cochrane review reported that, although both brief and intensive intervention improve cessation at the time of surgery, intensive intervention involving multiple sessions for at least 4 weeks before surgery had a stronger effect on cessation and improved both long-term cessation and all-cause complications (RR 0.42 ; 95\% CI $0.27-0.65$ ) and wound complications (RR $0.31 ; 95 \%$ CI 0.16-0.62) [25].

\section{Psychological prehabilitation}

Patients undergoing surgery may have psychosocial distress, which is related to the underlying diagnosis, the treatment and the disability. Psychosocial status including mood, anxiety, health beliefs and expectations, has been suggested to be associated with postoperative outcome [5]. Preoperative negative illness beliefs, depression, anxiety and low expectation of future health were associated with worse postoperative conditions such as functional recovery, pain and mortality. Therefore, psychological intervention before surgery would be an important trial to improve postoperative outcome. In particular, psychological factors can be modifiable by intervention, although the type and the degree of psychological factors are variable. For example, a trained psychologist can instruct each patient on relaxation techniques and breathing exercises to reduce anxiety [23, 24]. Patients practice with the psychologist during the initial visit and then an instructional $\mathrm{CD}$ is provided for performing the exercises at home until the operation.

Prevention of delirium and cognitive dysfunction after surgery is also an important strategy to improve postoperative functional recovery of patients. Although clinical data are limited, there is an interesting experimental report regarding the effects of prehabilitation on cognitive function after surgery. Kawano et al. [26] demonstrated that preoperative interventions with physical activity (a running wheel) and cognitive activity (a modified Hebb-Williams maze) for 14 days attenuated cognitive deficits and cytokine production in the brain after abdominal surgery in rats. Prehabilitation with exercise and cognitive intervention may be effective in preventing delirium and cognitive dysfunction.

\section{The efficacy of surgical prehabilitation}

Before surgery, in addition to optimizing comorbidities of the patients, physical, nutritional and psychological optimization has become an important part of perioperative management, especially in high-risk patients. Recent models of preoperative preparation use face-to-face multidisciplinary 
consultations to educate the patients on ways to improve physical activity, and nutritional and psychological status as well as how to stop smoking. These methods can also be used by patients after surgery to extend healthy life expectancy and are therefore termed 'surgical school' [5].

There have been several studies to evaluate the effects of multimodal prehabilitation programs as care package intervention-like enhanced recovery programs. Gillis et al. [27] evaluated a tri-modal prehabilitation program consisting of moderate intensity physical exercise combined with nutritional counselling, protein supplementation and anxiety reduction strategies, and indicated that $>80 \%$ of patients with prehabilitation returned to preoperative functional capacity by 8 weeks, while only $40 \%$ of patients without prehabilitation returned to baseline function. Moran et al. [28] conducted a systematic review and meta-analysis to assess the effects of prehabilitation on postoperative outcome after intra-abdominal surgery and demonstrated that prehabilitation consisting of inspiratory muscle training, aerobic exercise, and/or resistance training decreased postoperative complications (OR $0.59,95 \%$ CI $0.38-0.91$, $P=0.03$ ). However, recent evidence based on systematic reviews indicated that data regarding the efficacy of prehabilitation on clinical outcome remained too poor to reach any conclusion [29]. In particular, its efficacy can vary depending on the degree and type of interventions, the type of surgery and underlying conditions of the patients. Further prospective studies are warranted to identify the best prehabilitation program to improve the outcome.

\section{The role of PSH}

Recently, the concept of PSH has been advocated as a new model for the care of surgical patients [1,2]. The American Society of Anesthesiologists strongly supports the development of PSH as the future model of anesthesia practice. The PSH model is defined as a patient-centered and physician-led multidisciplinary and team-based system of coordinated care, which guides the patient throughout the entire surgical experience, from the time the surgeon decides to operate until 30 days post discharge. The central idea is to optimize the patient for surgery based on risk stratification and pre-developed evidence-based protocols, and improve the outcome at the lowest cost. Multidisciplinary teams consisting of anesthesiologists, surgeons, nurses, pharmacists, physical therapists, case managers, social workers and information technology experts are involved in PSH. Therefore, a preoperative program associated with prehabilitation can be applied by this team in PSH and possibly improve the patient's capacity before surgery and subsequently affect the outcome after surgery, including activities of daily living and quality of life.
In order to provide an adequate program for prehabilitation in $\mathrm{PSH}$, all the members involved in perioperative management should understand the importance of prehabilitation and commence the prehabilitation program using a multidisciplinary team approach as soon as the surgeon decides to operate. Occasionally, if the preoperative functional capacity of the patient is not appropriate to overcome the stress from the surgery, the team members may recommend postponing the surgery until the patient's functional capacity has improved; however, much data would be required on the efficacy of prehabilitation programs. Anesthesiologists are expected to play a role as a leader of this multidisciplinary team as 'perioperativists' [3].

\section{Summary}

In this review, we have described the importance of prehabilitation programs. In an increasingly aged population, improvement of preoperative functional capacity is crucial to provide better recovery and quality of life after surgery. Anesthesiologists should be involved in the perioperative management as perioperativists to optimize the patient's conditions to overcome the surgical stress. Further investigation on prehabilitation and the development of guidelines for prehabilitation programs are warranted.

\section{References}

1. Kain ZN, Vakharia S, Garson L, Engwall S, Schwarzkopf R, Gupta R, Cannesson M. The perioperative surgical home as a future perioperative practice model. Anesth Analg. 2014;118(5):1126-30.

2. Desebbe O, Lanz T, Kain Z, Cannesson M. The perioperative surgical home: an innovative, patient-centred and cost-effective perioperative care model. Anaesth Crit Care Pain Med. 2016;35(1):59-66.

3. Kawaguchi M, Tanaka Y, Furuya H. Improving the safety and quality of surgical patient care: what can we learn from quality management of industries? J Anesth. 2015;29(4):485-6.

4. Tanaka Y, Yoshimura A, Tagawa K, Shida D, Kawaguchi M. Use of quality of recovery score (QoR40) in the assessment of postoperative recovery and evaluation of enhanced recovery after surgery protocols. J Anesth. 2014;28(1):156-9.

5. Levett DZ, Edwards M, Grocott M, Mythen M. Preparing the patient for surgery to improve outcomes. Best Pract Res Clin Anaesthesiol. 2016;30(2):145-57.

6. Carli F, Silver JK, Feldman LS, McKee A, Gilman S, Gillis C, Scheede-Bergdahl C, Gamsa A, Stout N, Hirsch B. Surgical prehabilitation in patients with cancer: state-of-the-science and recommendations for future research from a panel of subject matter experts. Phys Med Rehabil Clin N Am. 2017;28(1):49-64.

7. Tokudome S, Hashimoto S, Igata A. Life expectancy and healthy life expectancy of Japan: the fastest graying society in the world. BMC Res Notes. 2016;9(1):482.

8. Akai M, Doi T, Seichi A, Okuma Y, Ogata T, Iwaya T. Locomotive syndrome: operational definition based on a questionnaire, 
and exercise interventions on mobility dysfunction in elderly people. Clinic Rev Bone Metab. 2016;14:119-130.

9. Nakamura K, Ogata T. Locomotive syndrome: definition and management. Clin Rev Bone Miner Metab. 2016;14:56-67.

10. Yoshimura Nakamura K. Epidemiology of locomotive organ disorders and symptoms: an estimation using the population-based cohorts in Japan. Clin Rev Bone Miner Metab. 2016;14:68-73.

11. Shinkai S, Yoshida H, Taniguchi Y, Murayama H, Nishi M, Amano H, Nofuji Y, Seino S, Fujiwara Y. Public health approach to preventing frailty in the community and its effect on healthy aging in Japan. Geriatr Gerontol Int. 2016;16(Suppl 1):87-97.

12. Singh $M$, Stewart $R$, White $H$. Importance of frailty in patients with cardiovascular disease. Eur Heart J. 2014;35(26):1726-31.

13. Matsumoto H, Hagino H, Wada T, Kobayashi E. Locomotive syndrome presents a risk for falls and fractures in the elderly Japanese population. Osteoporos Sarcopenia. 2016;2:156-63.

14. Rizzuto D, Orsini N, Qiu C, Wang HX, Fratiglioni L. Lifestyle, social factors, and survival after age 75: population based study. BMJ. 2012;345:e5568.

15. Christensen T, Kehlet H. Postoperative fatigue. World J Surg. 1993;17:220-5.

16. Goto T, Maekawa K. Cerebral dysfunction after coronary artery bypass surgery. J Anesth. 2014;28(2):242-8.

17. Saito S. Management of cognition as reported in Japanese historical documents and modern anesthesiology research papers. J Anesth. 2016;30:739-44.

18. Wilson RJ, Davies S, Yates D, Redman J, Stone M. Impaired functional capacity is associated with all-cause mortality after major elective intra-abdominal surgery. $\mathrm{Br} \mathrm{J}$ Anaesth. 2010;105(3):297-303.

19. Levett DZ, Grocott MP. Cardiopulmonary exercise testing for risk prediction in major abdominal surgery. Anesthesiol Clin. 2015;33(1):1-16.

20. Katsura M, Kuriyama A, Takeshima T, Fukuhara S, Furukawa TA. Preoperative inspiratory muscle training for postoperative pulmonary complications in adults undergoing cardiac and major abdominal surgery. Cochrane Database Syst Rev. 2015;(10):CD010356.

21. Kushi LH, Doyle C, McCullough M, Rock CL, Demark-Wahnefried W, Bandera EV, Gapstur S, Patel AV, Andrews K, Gansler T, American Cancer Society 2010 Nutrition and Physical
Activity Guidelines Advisory Committee. American Cancer Society Guidelines on nutrition and physical activity for cancer prevention: reducing the risk of cancer with healthy food choices and physical activity. CA Cancer J Clin. 2012;62(1):30-67.

22. Chen BP, Awasthi R, Sweet SN, Minnella EM, Bergdahl A, Santa Mina D, Carli F, Scheede-Bergdahl C. Four-week prehabilitation program is sufficient to modify exercise behaviors and improve preoperative functional walking capacity in patients with colorectal cancer. Support Care Cancer. 2017;25(1):33-40.

23. Minnella EM, Awasthi R, Gillis C, Fiore JF Jr, Liberman AS, Charlebois P, Stein B, Bousquet-Dion G, Feldman LS, Carli F. Patients with poor baseline walking capacity are most likely to improve their functional status with multimodal prehabilitation. Surgery. 160(4):1070-9.

24. Weimann A, Braga M, Harsanyi L, Laviano A, Ljungqvist O, Soeters P, DGEM (German Society for Nutritional Medicine), Jauch KW, Kemen M, Hiesmayr JM, Horbach T, Kuse ER, Vestweber KH, ESPEN (European Society for Parenteral and Enteral Nutrition). ESPEN guidelines on enteral nutrition: surgery including organ transplantation. Clin Nutr. 2006;25(2):224-44.

25. Thomsen T, Villebro N, Møller AM. Interventions for preoperative smoking cessation. Cochrane Database Syst Rev. 2014;(3):CD002294.

26. Kawano T, Eguchi S, Iwata H, Tamura T, Kumagai N, Yokoyama M. Impact of preoperative environmental enrichment on prevention of development of cognitive impairment following abdominal surgery in a rat model. Anesthesiology. 2015;123:160-70.

27. Gillis C, Li C, Lee L, Awasthi R, Augustin B, Gamsa A, Liberman AS, Stein B, Charlebois P, Feldman LS, Carli F. Prehabilitation versus rehabilitation: a randomized control trial in patients undergoing colorectal resection for cancer. Anesthesiology. 2014;121(5):937-47.

28. Moran J, Guinan E, McCormick P, Larkin J, Mockler D, Hussey $\mathrm{J}$, Moriarty J, Wilson F. The ability of prehabilitation to influence postoperative outcome after intra-abdominal operation: a systematic review and meta-analysis. Surgery. 2016;160(5):1189-201.

29. Cabilan CJ, Hines S, Munday J. The impact of prehabilitation on postoperative functional status, healthcare utilization, pain, and quality of life: a systematic review. Orthop Nurs. 2016;35(4):224-37. 